\title{
AN OVERVIEW OF THE BOLTZMANN TRANSPORT EQUATION SOLUTION FOR NEUTRONS, PHOTONS AND ELECTRONS IN CARTESIAN GEOMETRY
}

\author{
Bárbara D. do Amaral Rodriguez ${ }^{1,3}$, Marco Túllio Vilhena ${ }^{2}$ \\ ${ }^{1}$ Programa de Pós-Graduação em Engenharia Mecânica \\ Universidade Federal do Rio Grande do Sul \\ Av. Sarmento Leite 425 sala 314 \\ 90230-181 Porto Alegre, RS \\ barbara.arodriguez@gmail.com \\ ${ }^{2}$ Departamento de Matemática Pura e Aplicada \\ Universidade Federal do Rio Grande do Sul \\ Av. Bento Gonçalves 9500 \\ 91509-900 Porto Alegre, RS \\ vilhena@mat.ufrgs.br \\ ${ }^{3}$ Instituto de Matemática, Estatística e Física (IMEF - FURG) \\ Universidade Federal do Rio Grande \\ Av. Itália, km 8, Campus Carreiros, Prédio do IMEF, sala 17 \\ 96201-900, Rio Grande, RS
}

\begin{abstract}
Questions regarding accuracy and efficiency of deterministic transport methods are still on our mind today, even with modern supercomputers. The most versatile and widely used deterministic methods are the $\mathrm{P}_{N}$ approximation, the $\mathrm{S}_{N}$ method (discrete ordinates method) and their variants. In the discrete ordinates $\left(\mathrm{S}_{N}\right)$ formulations of the transport equation, it is assumed that the linearized Boltzmann equation only holds for a set of distinct numerical values of the direction-of-motion variables. In this work, looking forward to confirm the capabilities of deterministic methods in obtaining accurate results, we present a general overview of deterministic methods to solve the Boltzmann transport equation for neutral and charged particles. First, we describe a review in the Laplace transform technique applied to $S_{N}$ two dimensional transport equation in a rectangular domain considering Compton scattering. Next, we solved the Fokker-Planck (FP) equation, an alternative approach for the Boltzmann transport equation, assuming a monoenergetic electron beam in a rectangular domain. The main idea relies on applying the $\mathrm{P}_{N}$ approximation, a recent advance in the class of deterministic methods, in the angular variable, to the two dimensional Fokker-Planck equation and then applying the Laplace Transform in the spatial $x$-variable. Numerical results are given to illustrate the accuracy of deterministic methods presented.
\end{abstract}

\section{INTRODUCTION}

The Boltzmann equation is an integro-differential equation representing a wide range of transport problems from astrophysics to traffic low [1]. Elegant analytical and numerical techniques have been developed to solve the Boltzmann equation for a broad class of transport and radiative transfer problems. These methods follow two distinct schools of thought: the probabilistic school, such as the Monte Carlo methods, which basic philosophy is to solve approximately the exact problem, and the deterministic school, such as the discrete ordinates methods, which basic philosophy is to solve exactly an approximate problem. The stochastic Monte Carlo method often is considered to be the ultimate numerical approach for radiation transport calculations, especially for complicated geometries. However if differential distributions are required, then a deterministic solution of the Boltzmann transport equation is often more efficient. 
Questions regarding accuracy and efficiency of deterministic transport methods are still on our mind today, even with modern supercomputers. The most versatile and widely used deterministic methods are the $\mathrm{P}_{N}$ approximation [2,3], the $\mathrm{S}_{N}$ method (discrete ordinates method) $[4,5]$ and their variants $[6,7]$. In the discrete ordinates $\left(S_{N}\right)$ formulations of the transport equation, it is assumed that the linearized Boltzmann equation only holds for a set of distinct numerical values of the direction-of-motion variables.

In the last decade, the $\mathrm{LTS}_{N}$ method was presented in the literature. This method solves, analytically, the discrete ordinates equation $\left(\mathrm{S}_{N}\right.$ equation) in a slab by the Laplace transform technique. The main idea comprehends the following steps: application of the Laplace transform technique to the set of the $S_{N}$ equations, solution of the resulting algebraic equation by the matrix diagonalization approach and inversion of the transformed angular flux by standard results of the Laplace transform theory. In earlier works $[6,8]$ the $\operatorname{LTS}_{N}$ method was applied in the solution of two dimensional transport equation assuming neutrons and photons in cartesian geometry.

On the other hand, in a recent work [9] we solved the Fokker-Planck (FP) equation, an alternative approach for the Boltzmann transport equation, assuming a monoenergetic electron beam in a rectangular domain. The Fokker-Planck (FP) approximation represents the impact of soft reactions as continuously slowing down the electrons, while also continuously changing their direction; e.g., a monodirectional beam will be dispersed into a finite beam width. This approximation can be derived from a Taylor series expansion of the integrand in the scatter source term appearing in the Boltzmann equation, with the assumption that only small changes in energy and direction are significant. The main idea relies on applying the $\mathrm{P}_{N}$ approximation, a recent advance in the class of deterministic methods, in the angular variable, to the two dimensional Fokker-Planck equation and then applying the Laplace Transform in the spatial $x$-variable. As a result, a first order linear differential equation in the spatial $y$-variable, is attained, which the solution is straightforward. The $\mathrm{P}_{N}$ approximation consists in expanding the angular variable of the angular flux in terms of the Legendre polynomials. Therefore, in this work, looking forward to confirm the capabilities of deterministic methods in obtaining accurate results, we present a general overview of deterministic methods to solve the Boltzmann transport equation for neutral and charged particles. Indeed, to reach our objectives, we organized this work as follows: in section 2 we present a review in the Laplace transform technique applied to $S_{N}$ two dimensional transport equation in a rectangular domain considering Compton scattering. In section 3 we describe in detail the two dimensional Fokker-Planck (FP) equation solution. In section 4 we report numerical simulations and comparisons. Concluding remarks and suggestions for future work are given in section 5 .

\section{THE LTS ${ }_{N}$ NODAL SOLUTION IN A RECTANGULAR DOMAIN}

Let us consider the two dimensional $\mathrm{S}_{N}$ nodal problem assuming Klein-Nishina scattering kernel and multigroup model [8]:

$$
\begin{array}{r}
\mu_{n} \frac{\partial}{\partial x} I_{j n}(x, y)+\eta_{n} \frac{\partial}{\partial y} I_{j n}(x, y)+\mu_{l j} I_{j n}(x, y)= \\
=\frac{\Delta}{3} \sum_{l=0}^{L} \frac{2 l+1}{2} \sum_{r=1}^{G} c_{r} \alpha k_{r j} P_{l}\left(1+\lambda_{r}-\lambda_{j}\right) P_{l}\left(\mu_{n}\right) \sum_{i=1}^{N} P_{l}\left(\mu_{i}\right) I_{r i}(x, y) \omega_{i}
\end{array}
$$


subject to vacuum boundary condition in a rectangle $0 \leq x \leq a$ and $0 \leq y \leq b$. Here $j=1: G$, $n=1: N, N=\frac{M(M+2)}{2}$ is the cardinality of the discrete ordinates set (number of discrete directions), $M$ represents the order of the angular quadrature, $G$ is the number of energy groups (wavelengths), $\mu_{l j}$ is the linear attenuation coefficient, $I_{j n}(x, y)=I\left(x, y, \lambda_{j}, \Omega_{n}\right)$ is the angular flux at the discrete direction $\Omega_{n}=\left(\mu_{n}, \eta_{n}\right)$ for the $j_{t h}$ group, $\omega_{i}$ are the Level Symetric Quadrature $\left(\mathrm{LQ}_{N}\right)$ weights and $k_{r j}=k\left(\lambda_{r}, \lambda_{j}\right)$ is the Klein-Nishina scattering kernel $[6,10]$.

It is important to mention that, in this work, the integral term in the wavelenght variable is approximated by the Simpson's rule [10].

To construct the $\mathrm{LTS}_{N}$ nodal solution for problem (1) we begin performing the transverse integration of this equation. This procedure yields to the set of the ensuing two coupled $S_{N}$ equations,

$$
\begin{array}{r}
\eta_{n} \frac{\partial}{\partial y} I_{j n y}(y)+\frac{\mu_{n}}{a}\left[I_{j n}(a, y)-I_{j n}(0, y)\right]+\mu_{l j} I_{j n y}(y)= \\
=\frac{\Delta}{3} \sum_{l=0}^{L} \frac{2 l+1}{2} \sum_{r=1}^{G} c_{r} \alpha k_{r j} P_{l}\left(1+\lambda_{r}-\lambda_{j}\right) P_{l}\left(\mu_{n}\right) \sum_{i=1}^{N} P_{l}\left(\mu_{i}\right) I_{r i y}(y) \omega_{i}
\end{array}
$$

for $j=1: G, n=1: N$. Here $I_{j n}(a, y)$ and $I_{j n}(0, y)$ are the angular fluxes exiting at the boundary and the average angular flux is written like,

$$
\begin{gathered}
I_{j n y}(y)=\frac{1}{a} \int_{0}^{a} I_{j n}(x, y) d x \\
\mu_{n} \frac{\partial}{\partial x} I_{j n x}(x)+\frac{\eta_{n}}{b}\left[I_{j n}(x, 0)-I_{j n}(x, b)\right]+\mu_{l j} I_{j n x}(x)= \\
=\frac{\Delta}{3} \sum_{l=0}^{L} \frac{2 l+1}{2} \sum_{r=1}^{G} c_{r} \alpha k_{r j} P_{l}\left(1+\lambda_{r}-\lambda_{j}\right) P_{l}\left(\mu_{n}\right) \sum_{i=1}^{N} P_{l}\left(\mu_{i}\right) I_{r i x}(x) \omega_{i},
\end{gathered}
$$

for $j=1: G, n=1: N$. Here $I_{j n}(x, b)$ and $I_{j n}(x, 0)$ are the angular fluxes exiting at the boundary and the average angular flux is written like,

$$
I_{j n x}(x)=\frac{1}{b} \int_{0}^{b} I_{j n}(x, y) d y .
$$

To this point we are in position to apply the LTS $_{N}$ method. Indeed, we begin applying the Laplace transform technique in equation (2). This procedure yields:

$$
\begin{aligned}
s \overline{I_{j n y}}(s)+\frac{\mu_{l j}}{\eta_{n}} \overline{I_{j n y}}(s)-\frac{\Delta}{3 \eta_{n}} \sum_{l=0}^{L} \frac{2 l+1}{2} \sum_{r=1}^{G} c_{r} \alpha k_{r j} P_{l}\left(1+\lambda_{r}-\lambda_{j}\right) P_{l}\left(\mu_{n}\right) \times \\
\times \sum_{i=1}^{N} P_{l}\left(\mu_{i}\right) \overline{I_{\text {riy }}}(s) \omega_{i}=I_{j n y}(0)-\frac{\mu_{n}}{a \eta_{n}}\left[\overline{I_{j n}}(a, s)-\overline{I_{j n}}(0, s)\right],
\end{aligned}
$$


for $j=1: G$ and $n=1: N$, which can be recast in matrix form like,

$$
\left(s I-B_{j n y}\right) \overline{I_{j n y}}(s)=I_{j n y}(0)+\overline{Z_{(j-1) y}}(s)+\overline{S_{j n y}}(s) .
$$

Here $\overline{I_{j n y}}(s)$ is the $N$ components of the angular flux Laplace transformed vector in $y$ variable and $I_{j n y}(0)$ is the $N$ components of the angular flux vector in $y$ variable at $y=0$. They have the form:

$$
\begin{aligned}
& \overline{I_{j n y}}(s)=\left[\begin{array}{llll}
\overline{I_{j 1 y}}(s) & \overline{I_{j 2 y}}(s) & \ldots & \overline{I_{j N y}}(s)
\end{array}\right]^{T}, \\
& I_{j n y}(0)=\left[\begin{array}{llll}
I_{j 1 y}(0) & I_{j 2 y}(0) & \ldots & I_{j N y}(0)
\end{array}\right]^{T} .
\end{aligned}
$$

On the other hand, the components of matrix $B_{j n y}$ are given by,

$$
b_{y}(p, q)=\left\{\begin{array}{cc}
-\frac{\mu_{l j}}{\eta_{p}}+\frac{\Delta}{3 \eta_{p}} \sum_{l=0}^{L} \frac{2 l+1}{2} c_{j} \alpha k_{j j} P_{l}\left(\mu_{p}\right) P_{l}\left(\mu_{p}\right) \omega_{q} & \text { se } p=q \\
\frac{\Delta}{3 \eta_{p}} \sum_{l=0}^{L} \frac{2 l+1}{2} c_{j} \alpha k_{j j} P_{l}\left(\mu_{p}\right) P_{l}\left(\mu_{q}\right) \omega_{q} & \text { se } p \neq q
\end{array}\right.
$$

and the scattering term reads like,

$$
\overline{Z_{(j-1) y}}(s)=\sum_{i=1}^{j-1} H_{i y} \overline{I_{i n y}}(s)
$$

where the entries of constant matrix $H_{i y}$ are written like,

$$
h_{y}(p, q)= \begin{cases}\frac{\Delta}{3 \eta_{p}} \sum_{l=0}^{L} \frac{2 l+1}{2} c_{i} \alpha k_{i j} P_{l}\left(1+\lambda_{i}-\lambda_{j}\right) P_{l}\left(\mu_{p}\right) P_{l}\left(\mu_{p}\right) \omega_{q} & \text { se } p=q \\ -\frac{\Delta}{3 \eta_{p}} \sum_{l=0}^{L} \frac{2 l+1}{2} c_{i} \alpha k_{i j} P_{l}\left(1+\lambda_{i}-\lambda_{j}\right) P_{l}\left(\mu_{p}\right) P_{l}\left(\mu_{q}\right) \omega_{q} & \text { se } p \neq q .\end{cases}
$$

The vector $\overline{S_{j n y}}(s)$ has the generic component:

$$
\overline{S_{j i y}}(s)=-\frac{\mu_{i}}{a \eta_{i}}\left[\overline{I_{j i}}(a, s)-\overline{I_{j i}}(0, s)\right] .
$$

Similar procedure in the $x$ variable leads to the ensuing linear algebraic system,

$$
\begin{aligned}
s \overline{I_{j n x}}(s)+\frac{\mu_{l j}}{\mu_{n}} & I_{j n x}(s)-\frac{\Delta}{3 \mu_{n}} \sum_{l=0}^{L} \frac{2 l+1}{2} \sum_{r=1}^{G} c_{r} \alpha k_{r j} P_{l}\left(1+\lambda_{r}-\lambda_{j}\right) P_{l}\left(\mu_{n}\right) \times \\
& \times \sum_{i=1}^{N} P_{l}\left(\mu_{i}\right) \overline{I_{r i x}}(s) \omega_{i}=I_{j n x}(0)-\frac{\eta_{n}}{b \mu_{n}}\left[\overline{I_{j n}}(s, b)-\overline{I_{j n}}(s, 0)\right],
\end{aligned}
$$

which again can be recast in the matrix form as,

$$
\left(s I-A_{j n x}\right) \overline{I_{j n x}}(s)=I_{j n x}(0)+\overline{Z_{(j-1) x}}(s)+\overline{S_{j n x}}(s) .
$$


Here $\overline{I_{j n x}(s)}$ is the $N$ components of the angular flux Laplace transformed vector in $x$ variable and $I_{j n x}(0)$ is the $N$ components of the angular flux vector in $x$ variable at $x=0$. They have the form:

$$
\begin{aligned}
& \overline{I_{j n x}(s)}=\left[\begin{array}{llll}
\overline{I_{j 1 x}(s)} & \overline{I_{j 2 x}(s)} & \ldots & \overline{I_{j N x}(s)}
\end{array}\right]^{T}, \\
& I_{j n x}(0)=\left[\begin{array}{llll}
I_{j 1 x}(0) & I_{j 2 x}(0) & \ldots & I_{j N x}(0)
\end{array}\right]^{T} .
\end{aligned}
$$

Bearing in mind that for the Klein-Nishina scattering kernel the wavelength ranges from $\lambda_{0}$ to $\lambda_{0}+2$ ( $\lambda_{0}$ is the wavelength of the slab incoming radiation), we discretize, without loss of generality, this interval in five sub-intervals, we mean five groups, with the main feature that the first group (group 1) corresponds to the sub-interval with shortest wavelength and higher energy and group 5, to the sub-interval with the longest wavelength and lowest energy.

Solving recursively equations (7) and (15) for increasing wavelength, ( $j$ from 1 to 5 ) due to the down-scattering, the $\operatorname{LTS}_{N}$ solution for these equations are given by

$$
\overline{I_{j n y}}(s)=\left(s I-B_{j n y}\right)^{-1}\left[I_{j n y}(0)+\overline{Z_{(j-1) y}}(s)+\overline{S_{j n y}}(s)\right]
$$

and

$$
\overline{I_{j n x}}(s)=\left(s I-A_{j n x}\right)^{-1}\left[I_{j n x}(0)+\overline{Z_{(j-1) x}}(s)+\overline{S_{j n x}}(s)\right] .
$$

Taking the Laplace inversion of the above ansatz we get,

$$
I_{j n y}(y)=\mathcal{L}^{-1}\left\{\left(s I-B_{j n y}\right)^{-1}\left[I_{j n y}(0)+\overline{Z_{(j-1) y}}(s)+\overline{S_{j n y}}(s)\right]\right\}
$$

and

$$
I_{j n x}(x)=\mathcal{L}^{-1}\left\{\left(s I-A_{j n x}\right)^{-1}\left[I_{j n x}(0)+\overline{Z_{(j-1) x}}(s)+\overline{S_{j n x}}(s)\right]\right\},
$$

which by the Heaviside expansion technique can be recast like [10],

$$
\begin{array}{r}
I_{j n y}(y)=\sum_{k=1}^{j n} \beta_{k} e^{s_{k} y} I_{j n y}(0)+Z_{(j-1) y}(y) * \mathcal{L}^{-1}\left\{\left(s I-B_{j n y}\right)^{-1}\right\}+ \\
+S_{j n y}(y) * \mathcal{L}^{-1}\left\{\left(s I-B_{j n y}\right)^{-1}\right\}
\end{array}
$$

and

$$
\begin{array}{r}
I_{j n x}(x)=\sum_{k=1}^{j n} \beta_{k} e^{s_{k} x} I_{j n x}(0)+Z_{(j-1) x}(x) * \mathcal{L}^{-1}\left\{\left(s I-A_{j n x}\right)^{-1}\right\}+ \\
+S_{j n x}(x) * \mathcal{L}^{-1}\left\{\left(s I-A_{j n x}\right)^{-1}\right\}
\end{array}
$$

Here star denotes convolution. To complete the solution we have to determine the unknown leakage angular fluxes at boundary namely $I_{j n}(x, 0), I_{j n}(0, y), I_{j n}(x, b)$ and $I_{j n}(a, y)$. Following the work of Hauser [8] which states that the exponential approximation gives the best 
results for the two dimensional $\mathrm{LTS}_{N}$ nodal solution for deep penetration problems, we assume the ensuing approximation for the leakage angular fluxes,

$$
\begin{aligned}
& I_{j n}(x, 0)=F_{j n} e^{-\operatorname{sign}\left(\mu_{n}\right) \Lambda x} \\
& I_{j n}(0, y)=G_{j n} e^{-\operatorname{sign}\left(\eta_{n}\right) \Lambda y} \\
& I_{j n}(x, b)=O_{j n} e^{-\operatorname{sign}\left(\mu_{n}\right) \Lambda x} \\
& I_{j n}(a, y)=P_{j n} e^{-\operatorname{sign}\left(\eta_{n}\right) \Lambda y}
\end{aligned}
$$

where $\operatorname{sign}(\mu)$ denotes the signal function:

$$
\operatorname{sign}(\mu)=\left\{\begin{array}{cc}
1 & \text { if } \mu>0 \\
-1 & \text { if } \mu>0
\end{array}\right.
$$

and $\Lambda$ represents the decay constant parameter, which has to be choice a priori. In this work, we assume $\Lambda$, likewise [8], as being the absorption cross section. The functions $\operatorname{sign}\left(\mu_{n}\right)$ and $\operatorname{sign}\left(\eta_{n}\right)$ which appear in the equations (24) - (27) guarantee that the approximated angular fluxes will decay for any discrete direction. Replacing (24) - (27) in equations (23) and (24) the $x$-averaged and $y$-averaged angular fluxes solutions are complete after the Laplace Transform inversion. Applying the boundary conditions, we determine the integration constants and consequently the two dimensional LTS $_{N}$ nodal solution is well determined.

\section{THE SOLUTION OF THE TWO DIMENSIONAL FOKKER-PLANCK EQUATION}

In order to determine the angular flux of electrons in a rectangular domain, let us consider the following two dimensional, time independent electron transport equation [6],

$$
\begin{array}{r}
\mu \frac{\partial \psi(x, y, \bar{\Omega}, E)}{\partial x}+\eta \frac{\partial \psi(x, y, \bar{\Omega}, E)}{\partial y}+\sigma_{t}(E) \psi(x, y, \bar{\Omega}, E)= \\
=\int d E^{\prime} \int_{4 \pi} d \bar{\Omega}^{\prime} \sigma_{s}\left(E^{\prime} \rightarrow E, \bar{\Omega}^{\prime} \cdot \bar{\Omega}\right) \psi\left(x, y, \bar{\Omega}^{\prime}, E^{\prime}\right)
\end{array}
$$

in a rectangle $0 \leq x \leq a$ and $0 \leq y \leq b$, subject to vacuum boundary conditions. Here the angular flux, denoted as $\psi(x, y, E, \bar{\Omega})$, represents the flux of particles at position $(x, y)$, with energy $E$ travelling in direction $\bar{\Omega}=(\mu, \eta)$. The quantity $\sigma_{s}$ in Eq. (29) is the differential scattering cross-section and is written as,

$$
\sigma_{s}\left(E, \mu_{0}\right)=\frac{\sigma_{t}(E) \eta^{*}\left(\eta^{*}+1\right)}{\pi\left(1+2 \eta^{*}-\mu_{0}^{2}\right)}
$$


where $\eta^{*}>0$ is a typically small constant called the screening parameter.

Here it is important to mention that, in this work we focus on screened Rutherford scattering. Screened Rutherford scattering is one of the simplest models of elastic scattering of electrons from nuclei taking into account the screening of the nuclei by atomic electrons. It is obtained from the Schrödinger equation in the first Born approximation, using an exponential factor in the potential to model the screening effect [11]. An approximate formula for the screening parameter is written as,

$$
\eta^{*}=\frac{h^{2} Z^{\frac{2}{3}}}{4\left(a_{H}\right)^{2}\left(m_{e} v\right)^{2}},
$$

where $Z$ denotes the atomic number of the nucleus, $m_{e} v$ is the (relativist) momentum of the electron that is being scattered, $\hbar$ is the Planck constant and $a_{H}$ is the Bohr radius.

We now assume that the scattering process is sufficiently peaked in the forward direction so that the Fokker-Planck scattering description [12] is appropriate. Thus, the Fokker-Planck approximation [13] to transport problem (29) is given by,

$$
\begin{array}{r}
\mu \frac{\partial \psi^{F P}(x, y, \bar{\Omega}, E)}{\partial x}+\eta \frac{\partial \psi^{F P}(x, y, \bar{\Omega}, E)}{\partial y}= \\
=\frac{\sigma_{t r}}{2} \frac{\partial}{\partial \mu}\left[\left(1-\mu^{2}\right) \frac{\partial}{\partial \mu}\right] \psi^{F P}(x, y, \bar{\Omega}, E),
\end{array}
$$

where $\psi^{F P}(x, y, \bar{\Omega}, E)$ represents the Fokker-Planck angular flux of particles at position $(x, y)$, with energy $E$ travelling in direction $\bar{\Omega}=(\mu, \eta)$ and the coefficient $\sigma_{t r}$ is called the transport cross-section and is defined as,

$$
\sigma_{t r}=2 \pi \int_{-1}^{1} \int_{0}^{1} \sigma_{s}\left(E, \mu_{0}\right)\left(1-\mu_{0}\right) d \mu_{0} d \eta .
$$

Multiplying the Fokker-Planck equation (32) by $P_{n}(\mu)$, integrating over $\mu$, and using a recursion formula [14], we came out with the following $\mathrm{P}_{N}$ equations:

$$
\begin{aligned}
\frac{n+1}{2 n+1} \frac{\partial}{\partial x} \psi_{n+1}^{F P}(x, y, E)+\frac{n}{2 n+1} \frac{\partial}{\partial x} \psi_{n-1}^{F P}(x, y, E) & +\frac{2 n+1}{2} \frac{\partial}{\partial y} \psi_{n}^{F P}(x, y, E) T_{n}= \\
& =\frac{\sigma_{t r}}{2}[-n(n+1)] \psi_{n}^{F P}(x, y, E),
\end{aligned}
$$

with the angular flux moments in discrete ordinates approximated by a quadrature formula as follows,

$$
\psi^{F P}(x, y, \bar{\Omega}, E)=\sum_{l=0}^{L} \frac{2 n+1}{2} \psi_{n}^{F P}(x, y, E) P_{n}(\mu)
$$

for $n=0, \ldots, N$, with $\psi_{N+1}^{F P}(x, y, E)=0$ in the $\mathrm{P}_{N}$ approximation and $T_{n}$ represented by an integral term, which can be analytic solved, written as,

$$
T_{n}=\int_{-1}^{1} \sqrt{\left(1-\mu^{2}\right)} P_{n}(\mu) P_{n+1}(\mu) d \mu
$$


Once applied the Laplace transform technique in equation (34) in the spatial variable $x$, we came out with the following linear algebraic system in the matrix form,

$$
A_{n}{\overline{\psi_{n}^{F P}}}^{\prime}(s, y, E)+B_{n}(s) \overline{\psi_{n}^{F P}}(s, y, E)-C_{n} \psi_{n}^{F P}(0, y, E)=0 .
$$

Here $\overline{\psi_{n}^{F P^{\prime}}}(s, y, E)$ is the $N$ components vector of the derivative of the angular flux Laplace transformed in the $x$ variable with respect to $y$ and is written as,

$$
{\overline{\psi_{n}^{F P}}}^{\prime}(s, y, E)=\left[{\overline{\psi_{0}^{F P}}}^{\prime}(s, y, E) \quad{\overline{\psi_{1}^{F P}}}^{\prime}(s, y, E) \quad \ldots \quad{\overline{\psi_{N}^{F P}}}^{\prime}(s, y, E)\right]^{T} .
$$

Here the column vector $\overline{\psi_{n}^{F P}}(s, y, E)$ is the $N$ components of the angular flux Laplace transformed vector in $x$ variable and $\psi_{n}^{F P}(0, y, E)$ is the $N$ components of the angular flux vector in $x$ variable at $x=0$. They have the form:

$$
\begin{aligned}
& \overline{\psi_{n}^{F P}}(s, y, E)=\left[\begin{array}{llll}
\overline{\psi_{0}^{F P}}(s, y, E) & \overline{\psi_{1}^{F P}}(s, y, E) & \ldots & \overline{\psi_{N}^{F P}}(s, y, E)
\end{array}\right]^{T}, \\
& \psi_{n}^{F P}(0, y, E)=\left[\begin{array}{llll}
\psi_{0}^{F P}(0, y, E) & \psi_{1}^{F P}(0, y, E) & \ldots & \psi_{N}^{F P}(0, y, E)
\end{array}\right]^{T} .
\end{aligned}
$$

On the other hand, the components of matrices $A_{n}, B_{n}(s)$, and $C_{n}$ are given, respectively, by

$$
\begin{gathered}
A_{n}=\left[\begin{array}{ccccccc}
1 T_{0} & 0 & 0 & 0 & \cdots & & 0 \\
0 & 9 T_{1} & 0 & 0 & \cdots & & 0 \\
0 & 0 & 25 T_{2} & 0 & \cdots & 0 \\
\vdots & \vdots & \vdots & \vdots & \ddots & & \vdots \\
0 & 0 & 0 & 0 & \cdots & (2 N+1)^{2} T_{N}
\end{array}\right], \\
B_{n}(s)=\left[\begin{array}{ccccccc}
0 & 2 s & 0 & 0 & \cdots & & 0 \\
2 s & 6 \sigma_{t r} & 4 s & 0 & \cdots & & 0 \\
0 & 4 s & 30 \sigma_{t r} & 6 s & \cdots & & \vdots \\
\vdots & \vdots & \vdots & \vdots & \ddots & & 2 N s \\
0 & 0 & 0 & \cdots & 2 N s & N(N+1)(2 N+1) \sigma_{t r}
\end{array}\right] \\
C_{n}=\left[\begin{array}{cccccc}
0 & 2 & 0 & 0 & \cdots & 0 \\
2 & 0 & 4 & 0 & \cdots & 0 \\
0 & 4 & 0 & 6 & \cdots & 0 \\
\vdots & \vdots & \vdots & \vdots & \ddots & \vdots \\
0 & 0 & \cdots & 2 N-2 & 0 & 2 N \\
0 & 0 & 0 & \cdots & 2 N & 0
\end{array}\right]
\end{gathered}
$$

where $\sigma_{t r}$ and $T_{n}$ are defined by Eq. (33) and Eq. (36) respectively.

The solution of equation (37) is given by,

$$
\overline{\psi_{n}^{F P}}(s, y, E)=c 1(s) \cdot e^{-\left[B_{n}(s) \cdot A_{n}^{-1}\right] y}+C_{n} \cdot\left[B_{n}(s)\right]^{-1} \cdot \psi_{n}^{F P}(0, y, E),
$$


where $c_{1}(s)$ is an arbitrary constant. In this problem we determine the $c_{1}(s)$ value, by applying the boundary and interface conditions. Due to the linear character of the inverse Laplace Transform operator, taking the Laplace inversion of the above ansatz we get,

$$
\begin{array}{r}
\psi_{n}^{F P}(x, y, E)=\mathcal{L}^{-1}\left\{c 1(s) \cdot e^{-\left[B_{n}(s) \cdot A_{n}^{-1}\right] y}\right\}+ \\
+C_{n} \cdot \mathcal{L}^{-1}\left\{\left[B_{n}(s)\right]^{-1}\right\} \cdot \psi_{n}^{F P}(0, y, E) .
\end{array}
$$

Once obtained the inverses matrices $A_{n}^{-1}$ and $B_{n}^{-1}(s)$, we calculate the inversion of the first term of the equation (42) by using the Laplace convolution property. Here, it is important to mention that the inverse matrix $B_{n}^{-1}(s)$ wasn't obtained analytically, due to the existence of the $s$ parameter, a non numeric parameter. Therefore, we opt to calculate the inverse Laplace transform numerically - in this work we apply the Gauss quadrature inversion method, $[15,16]$.

\section{NUMERICAL RESULTS}

In order to illustrate the aptness of the deterministic methods to solve the two dimensional transport equation for neutral and charged particles, in the sequel we report numerical simulations for the absorbed energy in rectangular domains with different dimensions and compositions. We considered a homogeneous rectangular domain composed by water, tissue or bone. We also assume a monoenergetic $(\mathrm{E}=1.25 \mathrm{MeV})$ and monodirectional photon beam incoming on the edge of a rectangle. In this study, the energy deposited by the secondary electrons, generated by the Compton Effect, will be considered. The remaining effects will not be taken into account. The numerical results encountered for absorbed energy are compared with the ones obtained by the program Geant 4 .

The data were simulated using the Geant4 (version 9.1) Monte Carlo program. Just recalling, Geant4 [17] is a toolkit for simulating the passage of particles through matter. It includes a complete range of functionality including tracking, geometry, physics models and hits. The computational universe considered in this work was a monoenergetic and monodirecional source incomming in the centre line of a volume. In that way, it was possible to prevent the lost of particles on the borders, "named borders effect". For each simulation $10^{6}$ histories were generated. In what follows, we present numerical results for the problems:

Problem 1: Let us consider a homogeneous rectangular domain, constituted by water $(Z / A=$ $0.55508, \rho=1 \mathrm{~g} / \mathrm{cm}^{3}$ ) and vacuum boundary condition.

In Tables 1 and 2 we present, respectively, the $\mathrm{LTS}_{8}$ Nodal and the $\mathrm{P}_{9}$ approximation numerical simulations for the absorbed energy in a homogeneous rectangular geometry composed by water and comparisons with the Geant 4 program results. Bearing in mind that Geant 4 program applies the Monte Carlo's technique, given a closer look to the results in Tables 1 and 2, we promptly realize a good coincidence. In fact, observing the results in Tables 1 and 2, we notice that the maximum discrepancy found is lower than $3 \%$ and $7 \%$, respectively. In Tables 1a and $2 \mathrm{a}$ we display the numerical convergence, respectively, of the $\operatorname{LTS}_{N}$ Nodal and the $\mathrm{P}_{N}$ 
Table 1. Absorbed energy [keV/photon emitted from the source] in a homogeneous rectangular domain composed by water

\begin{tabular}{|c|c|c|c|}
\hline & \multicolumn{3}{|c|}{ Water, liquid } \\
\hline Domain dimension & LTS $_{8}$ & Geant4 & Discrepancy \\
\hline $20 \mathrm{~cm} \times 10 \mathrm{~cm}$ & 0.00309 & 0.00315 & $1.9 \%$ \\
\hline $20 \mathrm{~cm} \times 20 \mathrm{~cm}$ & 0.00457 & 0.00468 & $2.3 \%$ \\
\hline $30 \mathrm{~cm} \times 40 \mathrm{~cm}$ & 0.00114 & 0.00116 & $1.7 \%$ \\
\hline
\end{tabular}

Table 1a. LTS $_{N}$ numerical convergence

\begin{tabular}{|c|c|}
\hline$N$ & $20 \mathrm{~cm} \times 20 \mathrm{~cm}$ \\
\hline 2 & 0.00359043 \\
\hline 4 & 0.00411992 \\
\hline 6 & 0.00446904 \\
\hline 8 & 0.00457042 \\
\hline
\end{tabular}

Table 2. Absorbed energy [keV/photon emitted from the source] by the free electron in a homogeneous rectangular domain composed by water

\begin{tabular}{|c|c|c|c|}
\hline & \multicolumn{3}{|c|}{ Water, liquid } \\
\hline Domain dimension & $\mathrm{P}_{9}$ & Geant4 & Discrepancy \\
\hline $20 \mathrm{~cm} \times 10 \mathrm{~cm}$ & 0.01845 & 0.01971 & $6.4 \%$ \\
\hline $20 \mathrm{~cm} \times 20 \mathrm{~cm}$ & 0.03379 & 0.03609 & $6.4 \%$ \\
\hline $30 \mathrm{~cm} \times 40 \mathrm{~cm}$ & 0.04581 & 0.04893 & $6.4 \%$ \\
\hline
\end{tabular}

Table 2a. $\mathbf{P}_{N}$ numerical convergence

\begin{tabular}{|c|c|}
\hline$N$ & $20 \mathrm{~cm} \times 20 \mathrm{~cm}$ \\
\hline 1 & 0.02590432 \\
\hline 3 & 0.03199219 \\
\hline 5 & 0.03252043 \\
\hline 7 & 0.03370622 \\
\hline 9 & 0.03378688 \\
\hline
\end{tabular}


approximation results, in a rectangular domain composed by water for increasing $N$. In fact, observing the results in Table 1a, for $N=6$ and $N=8$ we notice a coincidence of one significant digits. Here, it is important to mention that the unique approximation made along the derivation of the LTS $_{N}$ nodal solution was in the leakage angular flux at boundary. In Table 2a, for $N=7$ and $N=9$, we notice a coincidence of three significant digits.

Problem 2: To check the influence of the material density in the absorbed energy calculation, let us consider a rectangular domain composed by bone cortical $(Z / A=0.51478, \rho=1.92$ $\mathrm{g} / \mathrm{cm}^{3}$ ) and vacuum boundary condition.

Table 3. Absorbed energy [keV/photon emitted from the source] in a homogeneous rectangular domain composed by bone, cortical [18]

\begin{tabular}{|c|c|c|c|}
\hline & \multicolumn{3}{|c|}{ Bone, cortical (ICRU44) } \\
\hline Domain dimension & LTS $_{8}$ & Geant4 & Discrepancy \\
\hline $20 \mathrm{~cm} \times 10 \mathrm{~cm}$ & 0.05588 & 0.05781 & $3.3 \%$ \\
\hline $20 \mathrm{~cm} \times 20 \mathrm{~cm}$ & 0.09087 & 0.09487 & $3.4 \%$ \\
\hline $30 \mathrm{~cm} \times 40 \mathrm{~cm}$ & 0.15771 & 0.16375 & $3.7 \%$ \\
\hline
\end{tabular}

Table 4. Absorbed energy [keV/photon emitted from the source] by the free electron in a rectangular domain composed by bone, cortical [18]

\begin{tabular}{|c|c|c|c|}
\hline & \multicolumn{3}{|c|}{ Bone, cortical (ICRU44) } \\
\hline Domain dimension & $\mathrm{P}_{9}$ & Geant4 & Discrepancy \\
\hline $20 \mathrm{~cm} \times 10 \mathrm{~cm}$ & 0.83790 & 0.91244 & $8.2 \%$ \\
\hline $20 \mathrm{~cm} \times 20 \mathrm{~cm}$ & 0.79284 & 0.86380 & $8 . \%$ \\
\hline $30 \mathrm{~cm} \times 40 \mathrm{~cm}$ & 0.89218 & 0.97249 & $8.3 \%$ \\
\hline
\end{tabular}

In Tables 3 and 4 we present, respectively, the $\mathrm{LTS}_{8}$ Nodal and the $\mathrm{P}_{9}$ approximation numerical simulations for the absorbed energy in a rectangle composed, respectively, by bone cortical and tissue soft, and comparisons with the Geant 4 program results, where the maximum discrepancy found is lower than $4 \%$ and $9 \%$. Our numerical results demonstrate that, for higher density materials, other effects must be taken into account, because when the density is increases, the number of interaction increases as well as the possibility of other processes production involving secondary electrons. We must also mention that we have done all the $\operatorname{LTS}_{N}$ nodal calculations using an AMD Athlon $1700(1.4 \mathrm{GHz})$ microcomputer while the Geant4 results are obtained using a Pentium $4(1.7 \mathrm{GHz})$ microcomputer. Furthermore, the maximum computational time observed to generate all the results in each table was 30 minutes for both methods: LTS $_{N}$ nodal solution and Monte Carlo technique. 


\section{CONCLUSIONS}

In this work, we described the advances in a class of deterministic methods for the Boltzmann transport equation for monoenergetic problems in $x, y$-geometry. We must emphasize that the Fokker-Planck solution reported keeps the analytical feature, in the sense that no approximation is made along its derivation from the $\mathrm{P}_{N}$ equations, except for the round-off error. Regarding the topic of analyticity, the $\mathrm{LTS}_{N}$ nodal solution reported also keeps the analytical feature, in the sense that the unique approximation made along the derivation of the LTS $_{N}$ nodal solution was in the leakage angular flux at the boundary. We must also emphasize that we attained a good agreement with the Monte Carlo technique results with a small computational effort. Bearing in mind the good agreement between the results attained by the deterministic methods described with the ones of Geant 4 , we are confident to stress that these methods are promising to solve the two dimensional Boltzmann transport equation for neutral and charged particles. Finally, we focus our future attention to the issue of extending the Boltzmann transport solution for two dimensional problems in a heterogeneous rectangle.

\section{ACKNOWLEDGMENTS}

M. T. V. is gratefully indebted to CNPq (Conselho Nacional de Desenvolvimento Científico e Tecnológico), for the partial financial support to this work.

\section{REFERENCES}

[1] A. Badruzzaman, "Performance of three-dimensional nodal discrete-ordinates method", Progress in Nuclear Energy, 18, pp. 137-144 (1986).

[2] B. Davison, Neutron Transport Theory, Oxford University Press, Oxford (1957).

[3] C. F. Segatto, M. T. Vilhena, R. P. Pazos, "On the convergence of the spherical harmonics approximations", Nuclear Science and Engineering, 1, pp. 114-119 (2000).

[4] M. T. Vilhena, L. B. Barichello, "An analytical solution for the multigroup slab geometry discrete ordinates problems", Transport Theory and Statistical Physics, 24, pp. 13371339 (1995).

[5] M. T. Vilhena, C. F. Segatto, L. B. Barichello, "A particular solution for the Sn radiative transfer problems", J. quant. spectrosc. radiat. transfer., 4, pp. 467-469 (1995).

[6] B. D. A. Rodriguez, Methodology for obtaining a solution for the Boltzmann Transport equation considering Compton scaterring simulated by Klein-Nishina, Doctoral dissertation, Mechanic Engineering Program, PROMEC/UFRGS (2007).

[7] C. F. Segatto, M. T. Vilhena, "Extension of the $\mathrm{LTS}_{N}$ formulation for discrete ordinates problems without azimuthal symmetry", Annals of Nuclear Energy, 21, pp. 701-710 (1994).

[8] E. B. Hauser, R.P. Pazos and M.T. Vilhena, "An error bound estimate and convergence of the nodal-LTS ${ }_{N}$ solution in a rectangle", Annals of Nuclear Energy, 32, pp. 1146-1156 (2005).

[9] B. D. A. Rodriguez, M. T. Vilhena, V. Borges, G. Hoff, "A closed-form solution for the two-dimensional Fokker-Planck equation for electron transport", Annals of Nuclear Energy, 35, pp. 958-962 (2008). 
[10] B. D. A. Rodriguez, M. T. Vilhena, V. Borges, "The determination of the exposure buildup factor formulation in a slab using the $\mathrm{LTS}_{N}$ method", Kerntechnik, 71, pp.182184 (2006).

[11] L. Reimer,Scanning Electron Microscopy, Springer-Verlag, Berlin (1985).

[12] G. C. Pomraning, "Flux-limited diffusion and Fokker-Planck equations, Nuclear Science and Engineering, 85, pp.116-126 (1983).

[13] C. Börges and E. W. Larsen, "On the accuracy of the Fokker-Planck and Fermi pencil beam equations for charged particle transport", Medical Physics, 23, pp. 1749-1759 (1996).

[14] D. L. Kreider, An Introduction to Linear Analysis, Addison-Wesley Publishing Company, Inc., Massachusetts, USA (1966).

[15] A. H. Stroud and D. Secrest, Gaussian Quadrature Formulas, Prentice Hall Inc., New Jersey, USA (1986).

[16] B. Davies and B. Martin, "Numerical Inversion of the Laplace Transform: A Survey and Comparison of Methods", Journal of Computational Physics, 33, pp.1-32 (1979).

[17] S. Agostinelli et al., "Geant4 - A Simulation Toolkit", Nuclear Instruments and Methods A, 506, pp. 250-303 (2003).

[18] International Comission on Radiation Units and Measuremens, "Tissue Substitutes in Radiation Dosimetry and Measurement”, ICRU 44, Maryland, USA (1989). 